\title{
"Caught between a rock and a hard place: obesity and delivery decisions"
}

\author{
Mark Santillan, MD ${ }^{1}$
}

The incidence of obesity has achieved epidemic proportions. According to the CDC, $33.8 \%$ of all adults in the United States are obese. In 2008, obesity related costs in the US totaled $\$ 147$ billion. In addition to costs, obesity causes an increase in morbidity and mortality caused by heart disease, stroke, diabetes, and cancer. The increase in the proportion of women gaining more than 40 pounds during pregnancy and the related increased perinatal morbidity demonstrates how obesity has affected the reproductive outcomes for women. Obese women are more likely to develop diabetes, hypertension, preeclampsia, macrosomic babies, and the need for cesarean section. In addition to macrosomia, babies born to obese women are at increased risk for preterm mortality and congenital anomalies such as neural tube defects and cardiac defects. Offspring of obese women are also at increased risk for obesity, metabolic syndrome, hypertension, and behavioral disorders in adulthood.

Obesity provides additional challenges in antepartum testing and care. Non stress tests are not more likely to be nonreactive, but obese women are more likely to have more NSTs. Increasing $\mathrm{BMI}$ is correlated with increased failure rates and number of attempts to obtain a nuchal translucency measurement. Adequate second trimester anatomy scans are obtained at a much later gestation with increasing obesity. Failure rate for an adequate fetal heart examination is $20 \%$ in Class III obese women in comparison to $1.5 \%$ in nonobese women.

The increased morbidity and mortality in the obese woman challenges how we treat and counsel obese women during labor. In a dose response fashion, obese women are more likely to have a prolonged pregnancy requiring an induction of labor. Data also demonstrate that induction of labor of obese women is correlated with a higher need for oxytocin, longer time to delivery, higher cesarean delivery rate, and higher induction failure rates. The relationship between cesarean delivery rate and $\mathrm{BMI}$ is linear with a C-Section rate of $30 \%$ at the highest levels of obesity.

\section{${ }^{1}$ Department of Obstetrics and Gynecology, The University of lowa, lowa City, IA, 52240}

Please cite this paper as: Santillan M. "Caught between a rock and a hard place: obesity and delivery decisions." Proc Obstet Gynecol. 2011 November;2(2):Article 7 [ 2 p.]. Available from: http://ir.uiowa.edu/pog/. Free full text article.

Corresponding author: Mark Santillan, Department of Obstetrics and Gynecology, University of lowa, 31330 PFP, 200 Hawkins Drive, lowa City, IA, 52242. Telephone (319)353-7489, mark-santillan@uiowa.edu 
Yet, with the increased need for CSections in the obese, should primary C-sections be performed? Rates of morbidity and mortality are increased in this population. There are increased risks of wound infection/breakdown, need for more regional anesthesia attempts, and higher risk of general anesthesia. In some studies, when a planned vaginal delivery versus planned cesarean section for obesity is considered, there are no differences in morbidities and mortality between the two approaches. Yet, for women with a previous cesarean delivery who qualify for a VBAC, a trial of vaginal delivery is associated with a higher risk of composite maternal morbidity and composite neonatal injury. ACOG does not explicitly suggest either a primary vaginal or cesarean section in the obese population. Individualized counseling and preparation for the needs of an obese woman in labor are at the cornerstone of managing obese women in labor and delivery. 